\title{
VLBI and Broad-Band Radio Spectra Study of the Strongly Variable Extragalactic Object 0524+034
}

\author{
Y.Y. Kovalev ${ }^{1}$, A.G. Gorshkov², V.K. Konnikova ${ }^{2}$, M.G. Mingaliev ${ }^{3}$ \\ ${ }^{1}$ Astro Space Center of the Lebedev Physical Institute, \\ Profsoyuznaya 84/32, 117810 Moscow, Russia \\ ${ }^{2}$ Sternberg Astronomical Institute, Moscow, Russia \\ ${ }^{3}$ Special Astrophysical Observatory, N. Arkhyz, Russia
}

\begin{abstract}
Results of the first measurements of a VLBI structure of $0524+034$ at $6 \mathrm{~cm}$ at the EVN+HartRAO in 1999 and recent broad-band spectra monitoring data are reported. We suppose that precession of the relativistic jet can be one of the possible mechanisms to account for the observed milliarcsecond structure and the strong long-term variations of the spectrum. The detected rapid changes may be interpreted as a propagation of a shock in the inhomogeneous jet plasma, but interstellar scintillations can not be ruled out on the basis of available data.
\end{abstract}

\section{Introduction, Observations and Results}

The object $0524+034$ was first detected in the Zelenchuk survey in 1979, and later its flux was found to vary by a factor of more than ten with a characteristic time scale of several years (see also Figure 1). The source can be identified as a BL Lac (the optical spectrum contains no emission lines, Chavushyan et al. 2000). A flux density variability on a time scale less than 10 days was found in the simultaneous observations carried out with the RATAN-600 radio telescope in 1998 (Figure 1). The rapid flux density variations are correlated at all frequencies, at which the variability is revealed, including the optically thick part of the spectrum (Gorshkov et al. 2000). Here, we report the results of the first measurements of a VLBI structure of $0524+034$ with resolution of 1-2 mas. The source shows a compact, one-sided core-jet structure at a position angle of about $-90^{\circ}$ (Figure 1). An analysis of the derived multicomponent jet model indicates that the jet is probably beginning to curve at a distance of several mas from the VLBI core. More than $90 \%$ of the total emission of $0524+034$ is coming from the mas scale. The recent broad-band spectrum measurements were conducted nearly to and after the EVN experiment (for the method of observations and data reduction see Kovalev et al. 1999; Gorshkov et al. 2000). The rapid changes of the broad-band spectrum were confirmed, although the flux had dropped several times since 1998. The simultaneity of the rapid variations over a broad frequency range and the fact that the mean spectrum of the variable component coincides with the spectrum of a homogeneous, spherically symmetric source suggest the importance of acceleration of electrons and amplification of the magnetic field, whereas adiabatic expansion can be neglected. 


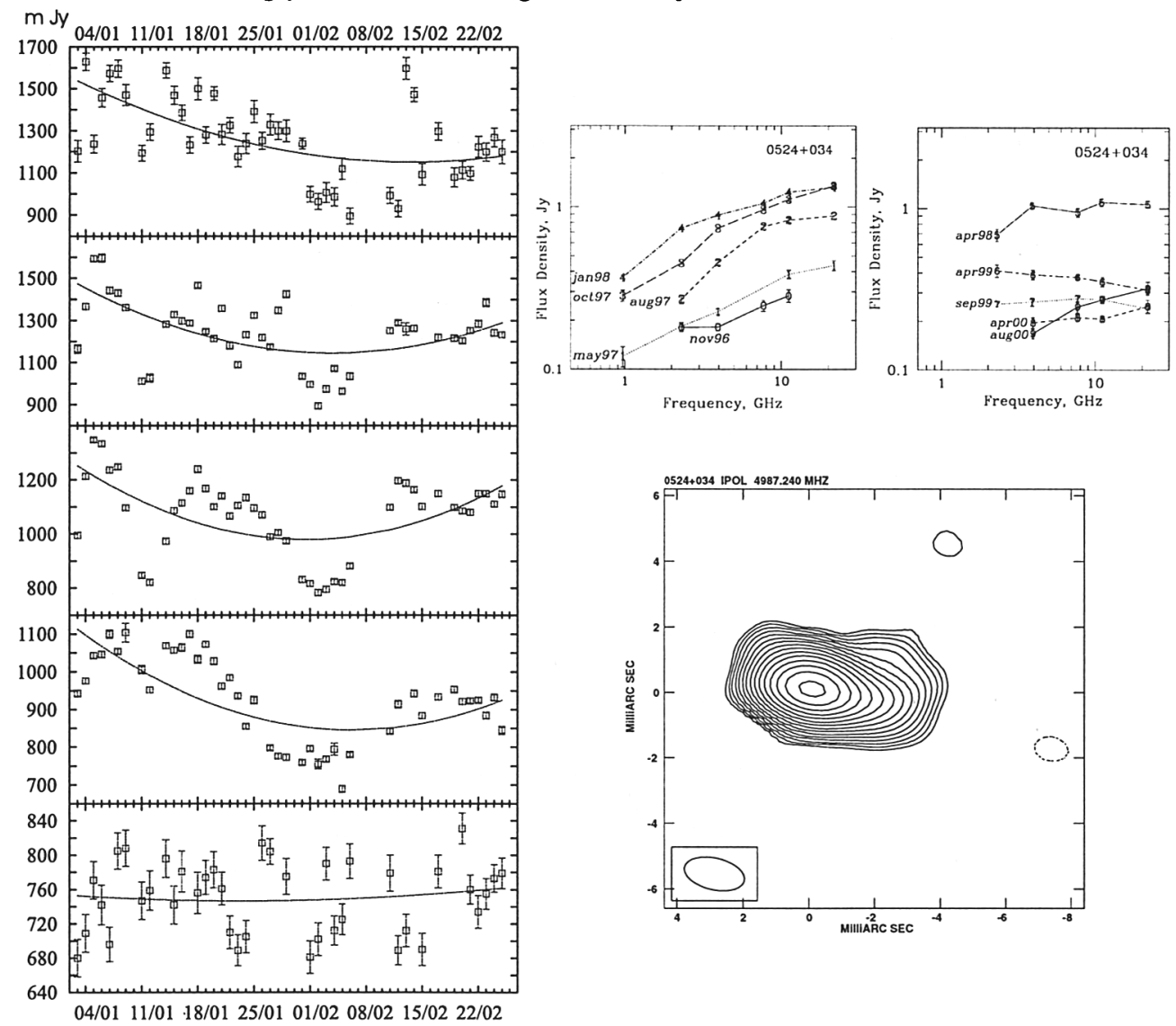

\section{$0524+034$}

Figure 1. Left: rapid flux density variability of $0524+034$ at 21.7, $11.1,7.7,3.9,2.3 \mathrm{GHz}$ (from top to bottom) in 1998. Right: long-term broad-band spectrum variability of $0524+034$ during the flare in 19962000 and total intensity EVN + HartRAO map of $0524+034$ at $6 \mathrm{~cm}$, epoch of 1999, February.

Acknowledgments. YYK is thankful to the IAU for the support of attending the General Assembly.

\section{References}

Chavushyan, V., et al., 2000, ALett, 26, 339

Gorshkov, A. G., Konnikova, V. K., Mingaliev, M. G., 2000, ARep, 44, 161

Kovalev, Y.Y., et al., 1999, A\&AS, 139, 545 